\title{
Evolución del comercio exterior del vino del Campo de Cariñena
}

\author{
Ma Concepción Estella Álvarez *
}

\begin{abstract}
RESUMEN
En este artículo se exponen las vicisitudes por las que ha atravesado el vino de Cariñena para su comercialización desde el siglo pasado. Comienza con el éxito y los altos precios del vino conseguidos por la demanda francesa, tras la invasión filoxérica del pais vecino,

favorecidos también por la inauguración del ferrocarril de vía estrecha Zaragoza-Cariñena y seguidos de la crisis de superproducción y consiguiente descenso de los precios tras finalizar el Tratado de Comercio con

Francia. Luego se explica la lenta recuperación a lo largo de este siglo, se facilitan las cantidades exportadas en el último cuarto del mismo, para analizar de forma más exhaustiva la situación del mercado exterior en la actualidad, donde el comercio europeo ha vuelto a ser el más importante y de modo especial el de la Unión Europea.

\section{ABSTRACT}

In this article the different mishaps that the Cariñena wine has undergone to be commercialized since the last century are shown. The study begins with the success and the high price of the wine, due to the French demand, after the phylloxera invasion in the neighbouring country, also favoured by the opening of the narrow-gauge railway Zaragoza-Cariñena, and followed by the superproduction crises and the subsequent fall of the prices after the end of the Commercial Treaty with France. Afterwards, we explain the slow recovery along this century, giving the exported quantities in the last quarter of the century, and then analyzing more thoroughly the situation of the foreign market nowadays, since the European trade has regained importance, specially the one in the European Union.

\footnotetext{
* Prof. Tutora del Centro Asociado de Madrid.
} 


\section{INTRODUCCIÓN}

El viñedo es el principal cultivo en el Campo de Cariñena con 21.000 Has. La propiedad está muy repartida, de ahí la importancia económica y social de la comercialización del vino para los agricultores de la comarca. Las dificultades para la exportación han sido enormes a lo largo de la historia por diferentes causas, a pesar de la fama del vino de Cariñena desde los tiempos de Felipe II, pero hasta el siglo pasado no se consiguió un mercado exterior importante.

Durante la segunda mitad del siglo XIX una serie de factores diversos contribuyeron a transformar muchas zonas rurales en regiones de monocultivo vitícola, aunque el hecho de mayor trascendencia fue la apertura del mercado francés a causa de la invasión filoxérica en sus viñedos. Debido a la demanda francesa, el precio del vino se multiplicó por tres y por cuatro en casi toda España. Esta euforia también se manifestó en Aragón; según la encuesta realizada por el Consejo Superior de Agricultura, Industria y Comercio, se refleja que la superficie de vid en 1877 era de $85.922 \mathrm{Ha}$. y en 1883 , de $143.438 \mathrm{Ha}$.

\section{EL MERCADO FRANCÉS}

En el Campo de Cariñena se plantaron gran cantidad de cepas a fines del s. XIX y el paisaje vitícola se incrementó considerablemente, puesto que los franceses encontraron aquí vinos de alta graduación alcohólica muy apropiados para mezclarlos con otros de menor grado ${ }^{1}$. Había comisionistas permanentes dispuestos a comprar cuanto se les ofreciera; a finales de 1879 las ventas realizadas en una semana fueron las que figuran en el Cuadro $n=1$ ?.

Por este motivo algunos almacenistas franceses, como la Casa Violet Frères, se instalaron en Cariñena y en Aguarón para comprar la cosecha de uva y vino a los viticultores de los pueblos de la comarca. En su bodega de Aguarón vinificaban entre 8.000 y 15.000 quintales. Solían elaborar vinos dulces, mistelas y, sobre todo, la piqueta ${ }^{3}$. Como consecuencia de

\footnotetext{
Ferrer Regales, M. (1957): El Campo de Cariñena. Zaragoza. Institución Fernando el Católico (C.S.I.C.), pág. 81

Gaceta Agrícola del Ministerio de Fomento. T. XIII, octubre-diciembre de 1879, págs. 632-3.

Era la preparación para el coupage. Llamaban asi los vinificadores españoles al producto final de las siguientes operaciones: una vez fermentada una cuba de uvas, sacaban el vino y sobre el orujo que quedaba en la vasija, echaban agua en cantidad conveniente; después se efectuaba una nueva fermentación y obtenian un vinillo de color muy claro, pero con más viveza de color que los vinagrillos franceses... Este vino agua era fermentado con brisas de ricas uvas, que
} 
todo esto, muchos agricultores de este municipio arrancaron los olivares y los sustituyeron por viñas.

Cuadro 1

\begin{tabular}{lrc}
\hline MUNICIPIOS & Alqueces $^{4}$ & Precio en reales $^{4}$ \\
\hline Aguarón & 3.000 & 104 \\
Almonacid & 1.500 & 100 \\
Cariñena & 20.000 & 110 \\
Cosuenda & 1.500 & 100 \\
Encinacorba & 1.300 & 110 \\
Longares & 900 & 100 \\
Paniza & 1.300 & 110 \\
\hline
\end{tabular}

Desde 1881 había empezado a funcionar el ferrocarril Madrid-Barcelona, por lo que Morata de Jalón, limítrofe a los términos municipales más occidentales del Campo de Cariñena, se convirtió en un gran centro vinícola desde donde el vino de la zona se distribuía a sus lugares de destino. Con el ferrocarril de vía estrecha Cariñena-Zaragoza, construido entre 1882 y 1885 e inaugurado en 1887 , se permitió que el vino alcanzara más fácilmente el mercado francés, previo enlace con la red Zaragoza-Alsasua-Irún ${ }^{5}$.

Pero esta euforia no duró demasiado, pues al finalizar el Tratado de Comercio con Francia la demanda francesa fue disminuyendo significativamente; en primer lugar incrementaron los aranceles, luego rebajaron la escala alcohólica: al principio nuestros vinos no podían sobrepasar los $14^{\circ}$, después bajaron a $12^{\circ} y$, por último, a 10우 finalmente, llegó la imposición de derechos, con lo cual la frontera se cerró definitivamente al vino español. Y a pesar de las salidas del vino de Cariñena con destino a Centroeuropa por el puerto de Valencia, no se consiguió recuperar la pérdida del mercado francés, según $A$. Sabio.

Esta crisis del viñedo fue muy grave y se produjo la primera oleada de emigración del campo a la ciudad. Aunque la Casa Violet Frères con-

ya habian dado un vino tinto de 16 a 18 grados y hacia las veces del vinagrillo francés... pues la piqueta resultaba con mayor fuerza que el vinillo francés.

LISO Y TORRES, J. (1895): Estudio de las ventajas e inconvenientes que ha de producir en las principales comarcas españolas vinicolas de España la reciente Ley sobre introducción de vinos franceses para el coupage. Juegos florales del 16 de octubre de 1894. Zaragoza, pág. 725.

4 Un alquez equivale a 12 cántaros y a 118,92 litros.

5 Sabio Alcuten, A. (1995): Viñedo y vino en el Campo de Cariñena: Los protagonistas de las transformaciones (1860-1930). Zaragoza. Centro de Estudios Darocenses. Institución «Fernando el Católico", pág. 41. 
tinuó hasta principios de siglo en Aguarón, los precios que pagaba eran muy bajos y la cantidad de vino que compraba era ínfima. Inmediatamente a la denuncia del Tratado el precio del alquez se redujo a 3,25 pta., cuando anteriormente esta cantidad se había multiplicado por diez ${ }^{6}$.

Como compensación a la pérdida de las exportaciones, se pensó que el mercado interior podría absorber gran parte de la producción vinícola, al menos para el vino corriente. Y también se recurrió a la destilación. El ferrocarril concedió un mayor protagonismo a los almacenistas locales y a los comerciantes de Zaragoza que empezaron a servir de intermediarios para la redistribución. Pero este vino transportado por vía férrea tenía que realizar transbordos en Zaragoza por el diferente ancho de vía, de ahí que los costes se incrementaran sobremanera.

La crisis definitiva se planteó con la llegada de la filoxera al Campo de Cariñena a principios de siglo. Sin embargo, las replantaciones fueron muy rápidas en esta comarca, probablemente porque las condiciones ecológicas tan favorables para la vid hacian difícil encontrar cultivos sustitutivos más rentables. Por otra parte, los beneficios acumulados durante los años anteriores a una filoxera tardía ayudan a explicar también la rápida reconstrucción del viñedo por autofinanciación en algunos casos. A partir de 1910, superado ya lo peor de la crisis de mévente en Francia, parte de la exportación del vino de la comarca se canalizó nuevamente hacia el país vecino ?.

En los años treinta el ferrocarril Zaragoza-Valencia permitió hacer los envíos al mercado francés directamente desde Cariñena. No se trataba de vino de calidad, sino para coupage o para destinarlo a fábricas alcoholeras, pues se podía exportar el vino a Francia a buen precio. La mayoría de los viticultores preferian beneficios rápidos con procedimientos antiguos que realizar inversiones fuertes para modernizar el sector. El negocio consistía en vender vino barato. Por tanto, la fácil comercialización del producto para coupage no estimuló los cambios para la modernización en los procedimientos de vinificación ${ }^{8}$.

Mientras las exportaciones a Francia se mantuvieron altas, muchos cosecheros encabezaron el vino vendido al país vecino con alcohol industrial para reforzarlo y para que soportase mejor el transporte y el paso del tiempo. Al encabezar estos vinos en origen, los bodegueros y cosecheros de Cariñena preferían el alcohol industrial, que era cinco veces más bara-

\footnotetext{
Ferrer Regales, M. (1957). Op. cit., pág. 82.

Sabio Alcuten, A. (1995). Op. cit., pág. 51.

Sabio Alcuten, A. (1995). Op. cit, pág. 42.
} 
to que el vínico. Este encabezamiento no molestaba a los importadores franceses que veían una forma de introducir alcohol en su país eludiendo el severo régimen fiscal a que estaba sometido el tráfico de alcohol ${ }^{9}$.

Durante la II República, la Federación Vitícola Aragonesa pedía que el Estado se hiciera cargo de toda la producción de alcohol industrial para regular su venta en el mercado y asegurar un precio remunerador para el vino. Según Joaquín Tejero, uno de los vitivinicultores del Campo, se trataba de que el Estado tuviera en su mano el grifo del alcohol industrial para abrirlo con arreglo a las necesidades del mercado y con vistas a la protección de la viticultura. Sin embargo, el gobierno presionado por los fabricantes de alcohol vínico y de alcohol industrial abría y cerraba el grifo de acuerdo con una política ecléctica ${ }^{10}$.

La inauguración de la Estación Enológica de Cariñena en 1932 significó un gran avance en cuanto a las técnicas de elaboración. Tras el paréntesis exportador de la Guerra de 1936, se volvió a una situación parecida a la de finales del siglo XIX. La producción había disminuido, los precios se habían incrementado y con la II Guerra Mundial, se intensificó la demanda francesa y también las nuevas plantaciones de cepas. Esta nueva expansión viticola llevó a una superproducción y por desgracia a una nueva crisis, según $M$. Ferrer.

A partir de los años cuarenta, la generalización del uso del camión como medio de transporte convirtió a la ciudad de Cariñena en el centro coordinador de la venta y exportación de los vinos de la zona. Este fenómeno coincidió con la construcción de enormes bodegas que se constituyeron en las grandes compradoras de uvas y vino del Campo a to largo de todo el año y que iniciaron pronto pequeños ensayos en la venta al exterior. Por ejemplo, la bodega de Balbino Lacosta desde 1945 inició las ventas en Europa con partidas a Suiza y Francia, sobre todo ${ }^{11}$.

En esta misma década se fundaron algunas de las Bodegas Cooperativas más importantes que fueron canalizando y comercializando mejor la cosecha de los pequeños y medianos viticultores, sometidos hasta entonces duramente a las leyes de la oferta y la demanda y que tímida y lentamente comenzaron a exportar unos años más tarde.

\footnotetext{
9 Piqueras, J. (1981): La vid y el vino en el Pais Valenciano. Valencia. Institució Alfons el Magnànim, p. 69 .

10 Sabio Alcuten, A. (1995). Op. cit., pág. 67.

$"$ Esta bodega tenía la ventaja de que poseía su propio apartadero de ferrocarril y en él preparaban un tren completo de vagones-cisterna.
} 


\section{LOS INICIOS DE LA DIVERSIFICACIÓN DEL MERCADO}

Debido a su alta graduación el vino de Cariñena ha tenido siempre dificultades en el mercado exterior. La mayor parte del vino exportado - excepto algunas cajas en botellas- se había vendido para coupage y, por tanto, a granel. Pero aún con todo, las Bodegas Cooperativas iniciaron poco a poco sus ventas al exterior a finales de los años sesenta. Así, la Cooperativa de San Valero de Cariñena realizaba un envio semanal a Andorra la Vella y a comienzos de los setenta había enviado un millón de botellas; de forma esporádica había comercializado pequeñas cantidades en Rhodesia, Venezuela, Suiza, Francia y Dinamarca. También la Cooperativa de San José de Aguarón exportaba a Andorra y Cariñena Vitícola vendió 15.000 litros en botellas y garrafas a Amberes. A principios de los setenta este mismo Grupo _ "Cariñena Vitícola”- comercializó vino embotellado por valor de 4 millones de pesetas a Suiza, Francia, Estados Unidos y Filipinas.

En cuanto a las bodegas particulares a principios de los setenta, Arvín había ido vendiendo pequeñas cantidades a Inglaterra, La Cariñenense comercializó en Alemania 2.400 botellas y la bodega de Vicente, Suso y Pérez exportó a Canadá, Estados Unidos, Alemania, Italia y Venezuela.

De vino a granel se comercializó bastante más cantidad fuera de España. La Sociedad Cariñena Vitícola en 1970 vendió 300.000 I. a Francia: 200.000 I. a Burdeos y 100.000 I. a Tarbes. En cuanto a las bodegas particulares, la de Balbino Lacosta exportó 300.000 I. a Alemania, Suiza y América del Sur, concretamente a Ecuador, Bolivia y Venezuela.

Pero la venta al exterior de vino a granel atravesó momentos muy difíciles, porque el Reglamento de la C.E.E. prohibía la importación de vinos para coupage y además, los vinos para el consumo humano directo tenían que poseer entre 8 y 15 grados con lo que la mayor parte del vino de Cariñena no tenía cabida en el consumo comunitario y había que intentar abrirse mercado en otros países. También se intentó con los miembros de la E.F.T.A., pero el acuerdo bilateral firmado entre Dinamarca y Portugal en el que se redujeron los derechos aduaneros de sus vinos, hizo que la diferencia de impuestos a favor de los vinos portugueses fuera de un $150 \%$ del valor de estos vinos en frontera, con lo que nuestro país quedaba fuera de cualquier competencia posible. Respecto al mercado sueco, las medidas del gobierno tendentes a frenar las importaciones (impuestos indirectos que gravan el consumo de vinos y espirituosos) incidian en su consumo y, por tanto, en el comercio de los países abastecedores del monopolio de bebidas sueco, entre los que se encontraba España. Por otra 
parte, los tratados comerciales firmados con los antiguos paises del Este (Bulgaria, Checoslovaquia, Hungría, Rumania y Yugoslavia) hacian prever alguna posibilidad para la exportación, pero había que tener en cuenta que algunos de ellos también cultivaban viñas. Con diversos países de Latinoamérica se habían firmado acuerdos comerciales; pero las dificultades de tipo político-económico por las que atravesaban estos países hacían un tanto aleatorio este mercado. En cambio el mercado norteamericano se miraba con optimismo por su elevada capacidad de consumo, aunque las ventas indirectas eran todavía muy reducidas ${ }^{12}$.

Sin embargo había que intentar un mayor esfuerzo en el mercado del vino embotellado, aunque estaba prácticamente en sus comienzos y tropezaba con grandes inconvenientes por su alta graduación. En los países del Mercado Común se consideraba licoroso el vino que sobrepasaba los 15 grados y tenía que pagar aranceles muy altos. Sólo de entrada los derechos aduaneros de protección de la C.E.E. eran de 6,30 pta./l. Dicha recaudación iba a un fondo para las primas a los agricultores y los envíos de sus productos a terceros países. Por tanto, había que hacer un gran esfuerzo en la exportación del vino en botellas, porque el vino embotellado y con denominación de origen obtenía un $210 \%$ más de ingresos. Además, había una desgravación de un $17 \%$ del valor y una compensación en alcohol ${ }^{13}$.

Todas las empresas exportadoras tenían que estar encuadradas en una de las seis cartas sectoriales que había en España ${ }^{14}$. De esta forma gozaban de amplios beneficios ${ }^{15}$. La mayoría de las del Campo de

12 Estella Álvarez, Ma C. (1982): Producción y comercialización del vino de Cariñena. Zaragoza. Institución Fernando el Católico (C.S.I.C.).

13 Por cada hectolitro de vino que se exportaba el estado daba una compensación de 15 pta. como una prima que no podía quedar reflejada, porque las primas con arreglo a la Comunidad es. taban prohibidas, pero todos los países las tenian y las ocultaban. En la campaña 1970-71 el F.O.R.P.P.A. pagó 90 pta./Hl. de vino exportado.

14 La Carta de Exportador al Sector de los vinos se estableció en 1969.

15 a) Aplicaciones del tipo vigente del Impuesto de compensación de gravámenes interiores como desgravación final a las exportaciones de vinos.

b) Posibilidad de concesión de crédito a la exportación con un porcentaje del $40 \%$.

c) Obtención en su caso de un coeficiente máximo de cobertura del $95 \%$ para riesgos políticos y extraordinarios, del $90 \%$ para riesgos comerciales y de elevación de costos y del $55 \%$ para prospección y asistencia a ferias.

d) Ayuda en la construcción de depósitos en puntos próximos a lugares de embarque y a la creación de stocks en el extranjero con unos porcentajes de crédito variables.

e) Prioridad para la realización de misiones comerciales del sector al extranjero. Prioridad al sector en la utilización de centros comerciales en el exterior dependientes de la Comisaria general de ferias y Promoción Comercial.

f) Concesión de otros tipos diversos de beneficios. 
Cariñena se incorporaron a NORTEXVIN (Grupo Norte de Exportadores de Vino) y algunas a la zona de Valencia. En cada grupo quedaban incluidas las firmas que voluntariamente lo decidian. Estos grupos de exportadores estaban constituidos por un mínimo de cinco empresas que hubieran exportado vino y se comprometieran a seguir haciéndolo en lo sucesivo por un valor mínimo de 135 millones de pesetas anuales ${ }^{16}$.

En abril de 1972 se publicó en el B.O.E. la Ley del Estatuto de la Viña, del Vino y de los Alcoholes, que junto con su reglamento constituian el marco legal donde se regulaban todas las actividades relacionadas con la producción, elaboración, circulación y comercio. Se planteaba en ella una reforma del viñedo cuyo objetivo fundamental era la mejora de la calidad.

Cuadro 2

\begin{tabular}{rr}
\hline Años & Hectolitros \\
\hline 1971 & 2.142 \\
1972 & 9.030 \\
1973 & 25.272 \\
1974 & 23.204 \\
1975 & 28.145 \\
1976 & 22.285 \\
1977 & 22.518 \\
1978 & 25.353 \\
1979 & 24.516 \\
1980 & 17.784 \\
1981 & 17.215 \\
1982 & 19.172 \\
1983 & 8.850 \\
1984 & 8.550 \\
1985 & 8.521 \\
1986 & 6.461 \\
1987 & 5.557 \\
1988 & 8.129 \\
1989 & 9.081 \\
1990 & 6.772 \\
1991 & 9.010 \\
1992 & 15.001 \\
1993 & 43.794 \\
1994 & 22.372 \\
1995 & 18.233 \\
1996 & 20.777 \\
1997 & 32.459 \\
1998 & 33.671 \\
\hline
\end{tabular}

h) Preferencia y prioridad en las autorizaciones a conceder por los Consejos reguladores cuando éstas sean requeridas por los exportadores.

16 Resumen del Sector Vitivinícola, 1970, págs. 84 y 85. 
A partir de 1973 las exportaciones del vino del Campo de Cariñena superaron los $20.000 \mathrm{HI}$. y se alcanzaron los $28.000 \mathrm{HI}$. en 1975 , como se observa en el cuadro número 2. Después de algunas cosechas excedentarias se planteó definitivamente la "crianza», pues en varias exposiciones y concursos los vinos de Cariñena preparados adecuadamente habian cosechado grandes éxitos ${ }^{17}$. En la década de los ochenta las ventas al exterior empezaron a descender y quedaron reducidas a la mitad desde 1983 para alcanzar el mínimo - 5.557 HI._ en 1987, como se puede observar en el gráfico número 1.

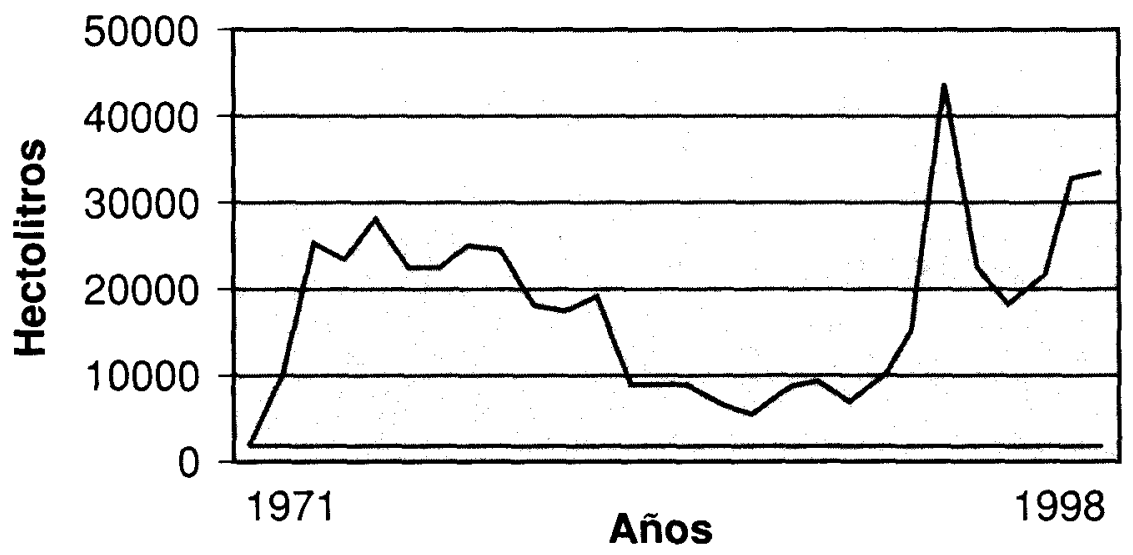

Gráfico 1. Evolución del vino de Cariñena exportado (1971-98).

La legislación comunitaria en materia de importación de vinos ponia en una situación muy complicada a los países terceros, entre los que se encontraba España y que vendía unos dos millones de hectolitros anuales. Con la firma del Tratado de Adhesión el 13 de julio de 1985 y la integración definitiva, a partir de Enero de 1986, se fijaron las condiciones específicas que van desde la aceptación del Compromiso de Dublín, que era la culminación de la Política Agraria Común (P.A.C.) en materia vitivinícola y dejaba sentadas las bases de esta política en dos aspectos fundamentales: $\left.1^{\circ}\right)$ Ordenar y regular el mercado del vino intentando adecuar la oferta y la demanda y desanimando a los viticultores que producian excedentes de vinos de mesa a través de una Destilación Obligatoria a precios

17 Estella Álvarez, Ma C. (1981): El viñedo en Aragón. Zaragoza. Institución Fernando el Católico (C.S.I.C.). 
cada vez más punitivos. $2^{\circ}$ ) Reconvertir y reestructurar el viñedo actual primando con ayudas monetarias el arranque definitivo de viñedos de vinos de mesa y los de las nuevas plantaciones sobre antiguas viñas de vino común, si se introducían variedades de uva «mejorantes» de la calidad. Todo ello para acabar con la producción de excedentes de vinos de mesa y potenciar los vinos de calidad cuya producción se comercializa mejor ${ }^{18}$. Esta política repercutió claramente en el Campo de Cariñena.

En la década de los noventa el comercio exterior se incrementó progresivamente para conseguir un máximo histórico en 1993 en que se alcanzaron los $43.794 \mathrm{HI}$. Pero desde que en 1994 la exportación se redujo un $50 \%$ se avanza paulatinamente en este ámbito del mercado exterior, puesto que la competencia es muy fuerte, tanto entre los vinos españoles como en los extranjeros y se está trabajando duramente para conseguir buena calidad a precios competitivos.

\section{LA SITUACIÓN ACTUAL}

En los dos últimos años en que la exportación ha vuelto a superar los $30.000 \mathrm{HI}$. se han obtenido premios y menciones honoríficas de gran renombre en los concursos vinícolas a nivel nacional e internacional.

El vino más demandado en el mercado exterior es el tinto, que supera el $90 \%$ del total, tanto joven como crianza y reserva. Se obtiene, sobre todo, a partir de la uva Garnacha negra, aunque en la actualidad se han conseguido muy buenos caldos con Tempranillo y Cabernet Sauvignon y no sólo como mejorantes, sino también como variedades únicas.

De blanco también se cultivan buenas viníferas como la Garnacha blanca y el Macabeo, especialmente en las zonas de mayor altura; se producen vinos excelentes e, incluso, cava, pero su exportación sólo alcanza el $8 \%$.

El rosado sólo se exporta en cantidades ínfimas, apenas el 1\%, como se aprecia en el gráfico número 2.

Para competir en el mercado exterior es necesario elaborar vino de calidad con las características propias de la zona, pero dándole un bouquet que agrade al paladar más exquisito. Por ello el propósito es exportar en botellas. En la actualidad el vino embotellado supera el $95 \%$ del total, según se aprecia en el gráfico número 3.

18 Piqueras Haba, J. (1985): La vitivinicultura valenciana ante la C.E.E. y el mercado mundial. Valencia. Conselleria d’ Agricultura, Pesca i Alimentatió. Generalitat Valenciana, págs. 35 y 123. 


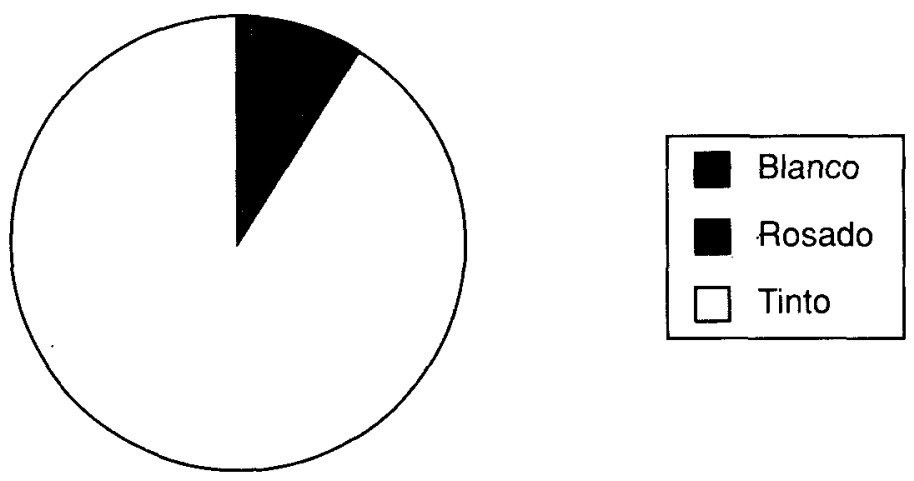

Gráfico 2. Vino de Cariñena embotellado y exportado en 1998.

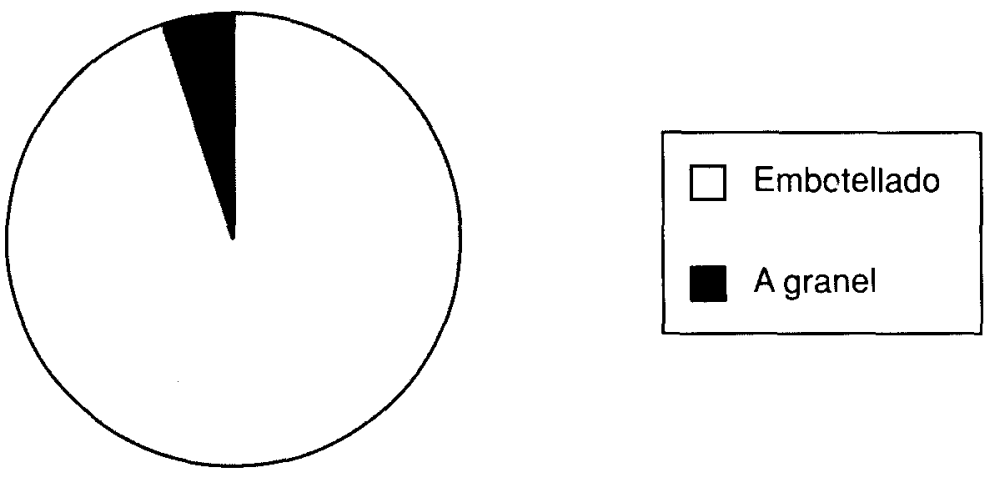

Gráfico 3. Vino de Cariñena exportado en 1998.

Como se puede observar en el gráfico número 4, con el vino embotellado se consigue una mayor rentabilidad económica, puesto que el vino en botellas que se ha comercializado en el exterior en 1998 ha obtenido el $97,5 \%$ del total en pesetas. En esta línea queda mucho por hacer. En los últimos años varias Bodegas Cooperativas del Campo de Cariñena se han unido para formar empresas mayores con el fin de comercializar mejor sus productos y junto con los enólogos están consiguiendo resultados muy positivos.

Entre los principales clientes del vino de Cariñena en 1998, corresponde el primer lugar al continente europeo con $31.880 \mathrm{HI}$., lo que significa el $94,7 \%$ del total exportado. Los países de la Unión Europea compraron 
$28.673 \mathrm{HI}$., lo que representa el $90 \%$ del total europeo y destaca el Reino Unido con $15.000 \mathrm{HI}$., seguido de lejos por Holanda con $3.164 \mathrm{HI}$., según se aprecia en el cuadro número 3 , mientras que los países no comunitarios europeos, incluida Rusia, apenas alcanzan el $10 \%$.
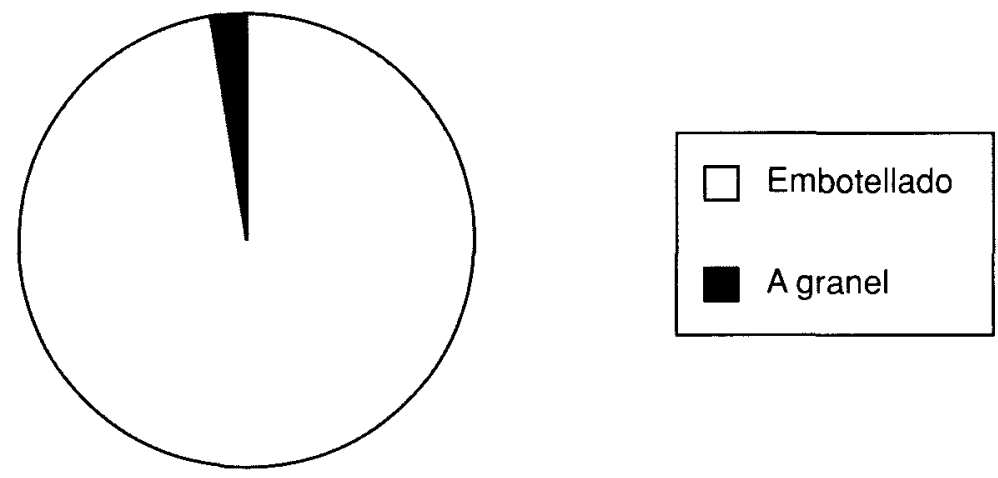

Gráfico 4. Valor del vino de Cariñena exportado en 1998 en Pesetas.

Cuadro 3

\begin{tabular}{lrrrr}
\hline PAISES & BLANCOS & ROSADOS & TINTOS & TOTAL \\
\hline ALEMANIA & 52 & 44 & 1.696 & 1.792 \\
BÉLGICA & 117 & 1 & 915 & 1.033 \\
DINAMARCA & 23 & 12 & 2.355 & 2.390 \\
FRANCIA & 1 & 28 & 738 & 767 \\
HOLANDA & 386 & 20 & 2.757 & 3.163 \\
R. IRLANDA & 277 & 0 & 522 & 799 \\
REINO UNIDO & 1.298 & 22 & 13.661 & 14.981 \\
ITALIA & 0 & 0 & 67 & 67 \\
LUXEMBURGO & 9 & 0 & 118 & 127 \\
ANDORRA & 0 & 0 & 87 & 87 \\
AUSTRIA & 1 & 0 & 52 & 53 \\
JAPÓN & 14 & 0 & 209 & 223 \\
SUECIA & 65 & 0 & 1.465 & 1.530 \\
SUIZA & 1 & 6 & 2.259 & 2.266 \\
U. S. A. & 5 & 0 & 143 & 148 \\
CANADÁ & 0 & 0 & 18 & 18 \\
VENEZUELA & 334 & 135 & 673 & 1.142 \\
AUSTRALIA & 0 & 0 & 108 & 108 \\
FINLANDIA & 0 & 0 & 1.323 & 1.323 \\
NORUEGA & 0 & 0 & 52 & 52 \\
RUSIA & 4 & 0 & 74 & 78 \\
TAIWAN & 0 & 0 & 92 & 92 \\
OMAN & 0 & 0 & 60 & 60 \\
& 2.587 & 268 & 29.444 & 32.299 \\
\hline
\end{tabular}


Las cantidades de vino importadas por el resto de los continentes son poco significativas. América compró sólo $1.310 \mathrm{HI}$., lo que representa el $3,9 \%$ del total y donde sobresale Venezuela con $1.142 \mathrm{HI}$., como se ve en el gráfico número 5. En Oceania, Australia adquirió $108 \mathrm{HI}$., lo que significa el $0,3 \%$ del total. En Asia, solamente Japón importó $223 \mathrm{Hl}$., seguido de Taiwan con $92 \mathrm{HI}$. y de Omán con $60 \mathrm{HI}$., lo que representa el $1,1 \%$ del total, según se observa en el gráfico número 6.

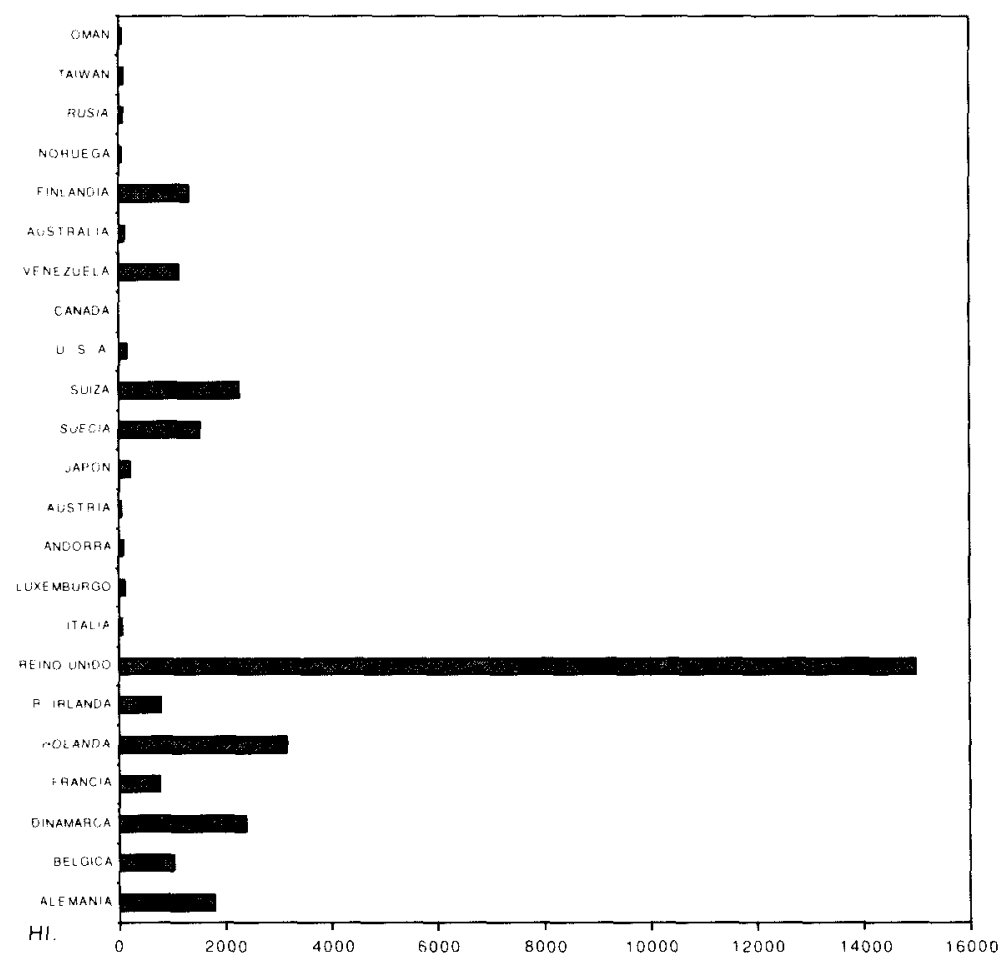

Gráfico 5. Exportación en botellas en 1998

\section{CONCLUSIONES}

Al finalizar este estudio y del análisis del mismo se pueden obtener las siguientes conclusiones:

1a. En el primer tercio del siglo XIX y principios del siglo XX el principal destino del vino de Cariñena fuera de España fue el mercado francés, después de la invasión filoxérica en este país y lo utilizaban para coupage. 

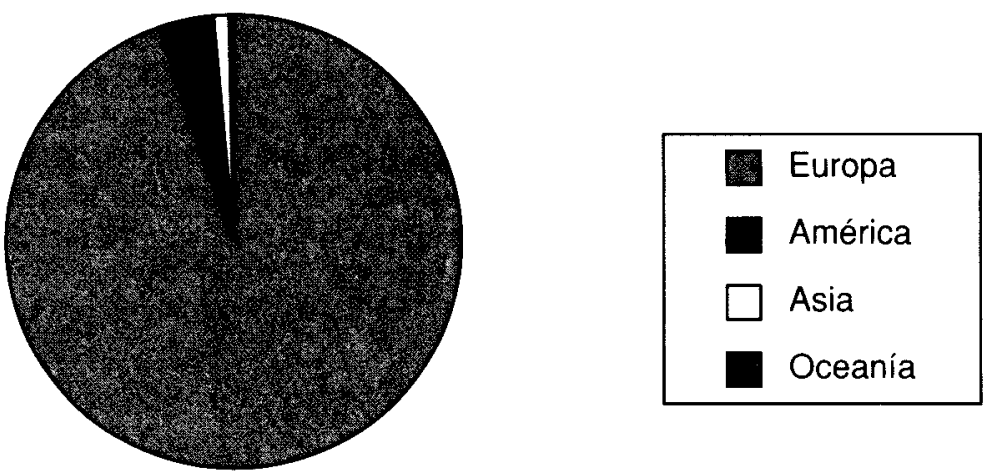

Gráfico 6. Vino de Cariñena exportado en 1998 por continentes.

$2^{\mathrm{a}}$. La exportación vinícola ocupa el primer lugar en el comercio exterior del Campo de Cariñena con un valor total de 867.593 .510 pesetas.

3a. El vino embotellado ha pasado a tener una gran importancia en el mercado internacional por la gran calidad que se ha conseguido en su elaboración, tanto en vino joven, como en Crianza, Reserva y Gran Reserva, aunque en el momento actual la mayor cantidad corresponde a vino joven.

4․ El tinto es el que más se produce y el que tiene mayor demanda, con más del $90 \%$ del total, seguido del blanco, con un $8 \%$ y del rosado con el $1 \%$, pues también su producción es bastante escasa.

$5^{a}$. El futuro hay que mirarlo con optimismo al suprimirse las barreras aduaneras que existian en los paises de la Unión Europea, aunque la competencia de algunos productores comunitarios y de muchas regiones españolas es muy fuerte y preocupante. Por este motivo tampoco es aconsejable descuidar los otros mercados extracomunitarios y extraeuropeos en los que sería muy conveniente incrementar las ventas.

\section{BIBLIOGRAFÍA}

Estella Alvarez, Mª C. (1982): Producción y comercialización del vino de Cariñena. Zaragoza. Institución Fernando el Católico (C.S.I.C.).

Estella Álvarez, M $\mathrm{M}^{z} \mathrm{C}$. (1981): El viñedo en Aragón. Zaragoza. Institución Fernando el Católico (C.S.I.C.).

Ferrer Regales, M. (1957): El Campo de Cariñena. Zaragoza. Institución Fernando el Católico (C.S.I.C.).

Frutos MEJiAs, L. Mà. (1982): El Campo en Aragón. Zaragoza. Colección Aragón. Librería General. 
Gaceta Agrícola del Ministerio de Fomento. T. XIII, octubre-diciembre de 1879.

Garrabou Segura, R. y Sanz Fernández, J. (1985): Historia Agraria de la España Contemporánea. 2. Expansión y crisis (1850-1900). Barcelona, Ed. Crítica.

LISO Y TORRES, J. (1895): Estudio de las ventajas e inconvenientes que ha de producir en las principales comarcas españolas vinícolas de España la reciente Ley sobre introducción de vinos franceses para el coupage. Juegos florales del 16 de octubre de 1894. Zaragoza.

NAREDO, J.MI. (1986): "La agricultura española en el desarrollo económico", en GaRRABou, R., Jiménez Blanco, J.I. (eds.): Historia Agraria de la España Contemporánea. 3. El fin de la agricultura tradicional (1900-1960). Barcelona, Ed. Crítica.

Pan-Montojo González, J. (1994): La bodega del mundo. La vid y el vino en España (19001936). Madrid, Ed. Alianza.

Pinilla NavarRo, V. (1992): "La producción agraria en Aragón (1850-1935)". Revista de Historia Económica, año $X, n^{\circ} 3$.

Piqueras Haba, J. (1981): La vid y el vino en el País Valenciano. Valencia. Institució Alfons el Magnànim.

PIQUeras HABA, J. (1985): La vitivinicultura valenciana ante la C.E.E. y el mercado mundial. Valencia. Conselleria d' Agricultura. Pesca i Alimentatió. Generalitat Valenciana.

Resumen del Sector Vitivinicola (1970).

SABIO AlCUTEN, A. (1995): Viñedo y vino en el Campo de Cariñena: Los protagonistas de las transformaciones (1860-1930). Zaragoza. Centro de Estudios Darocenses. Institución Fernando el Católico. 